\title{
Integrated Sensing and Warning Multifunctional Devices Based on the Combined Mechanical and Thermal Effect of Porous Graphene
}

Yexiong Huang, ${ }^{\dagger}$ Lu-Qi Tao, ${ }^{\ddagger}$ Jiabing Yu, Zeping Wang, ${ }^{\dagger}$ Congcong Zhu, ${ }^{\ddagger}$ Xianping Chen ${ }^{*},+, \neq$

${ }^{\dagger}$ College of Optoelectronic Engineering, Key Laboratory of Optoelectronic Technology and Systems, Ministry of Education, Chongqing University, Chongqing 400044, China.

${ }^{\ddagger}$ State Key Laboratory of Power Transmission Equipment \& System Security and New Technology, Chongqing University, Chongqing 400044, China. 
(a)
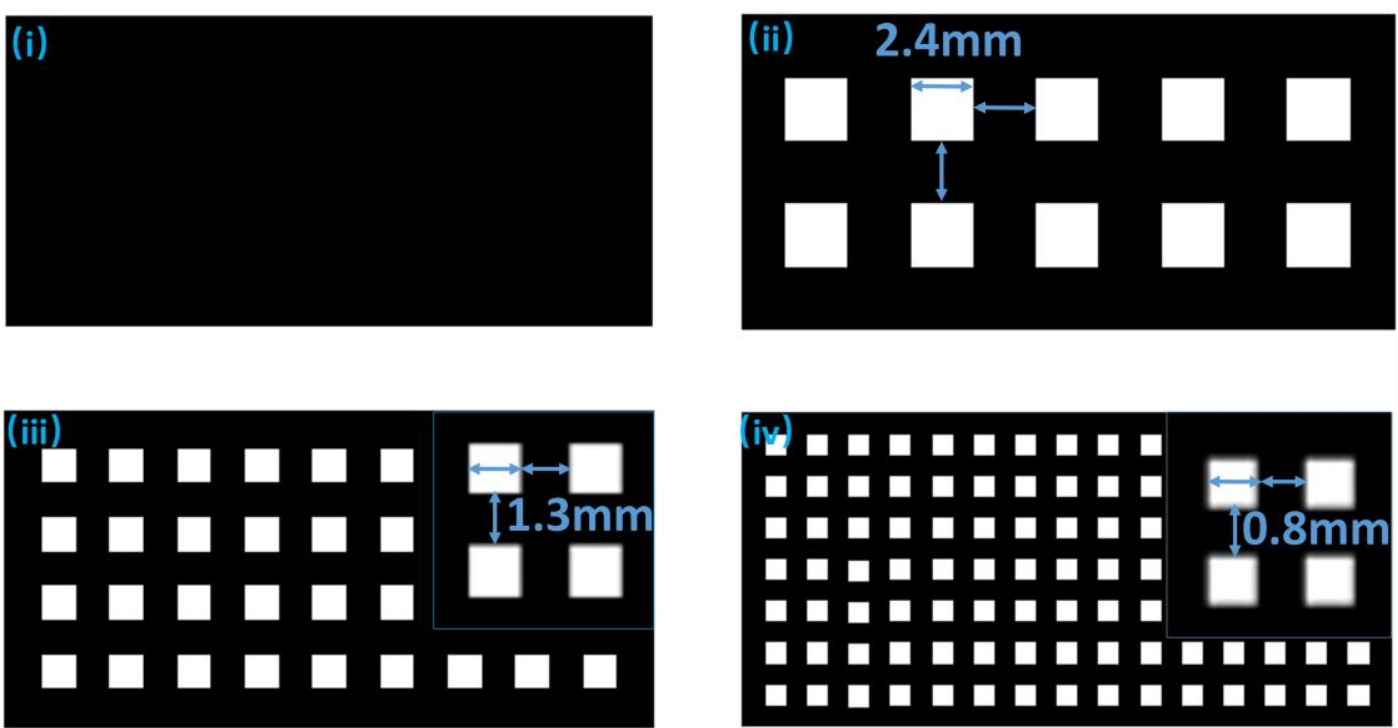

(b)

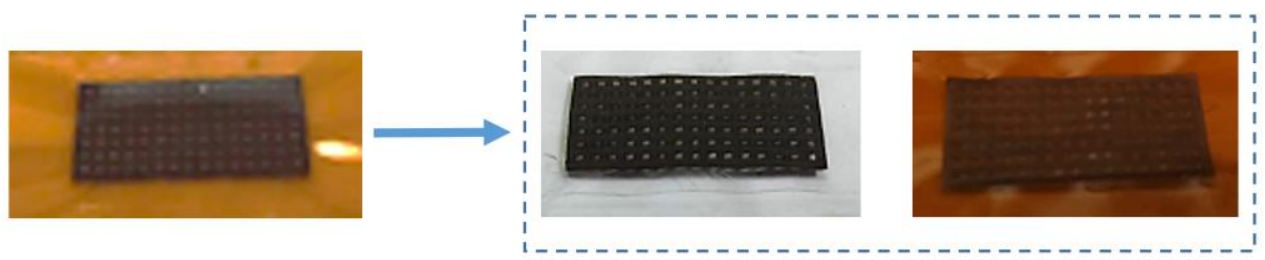

(c)
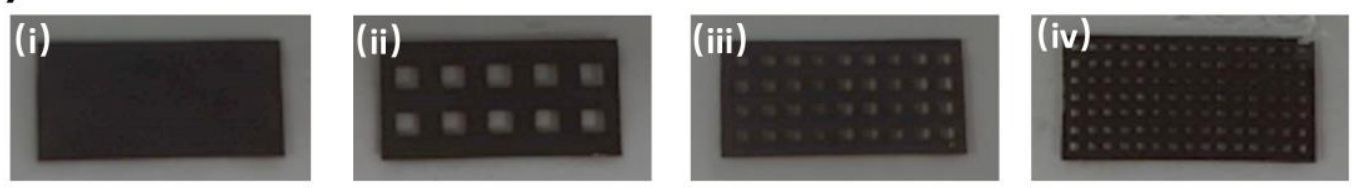

Figure S1. (a) The parameters of the mesh pattern for our device structures. (b) Digital images of the LIG before and after transfer from the PI to PDMS. (c) Digital images of our devices with different mesh patterns. 

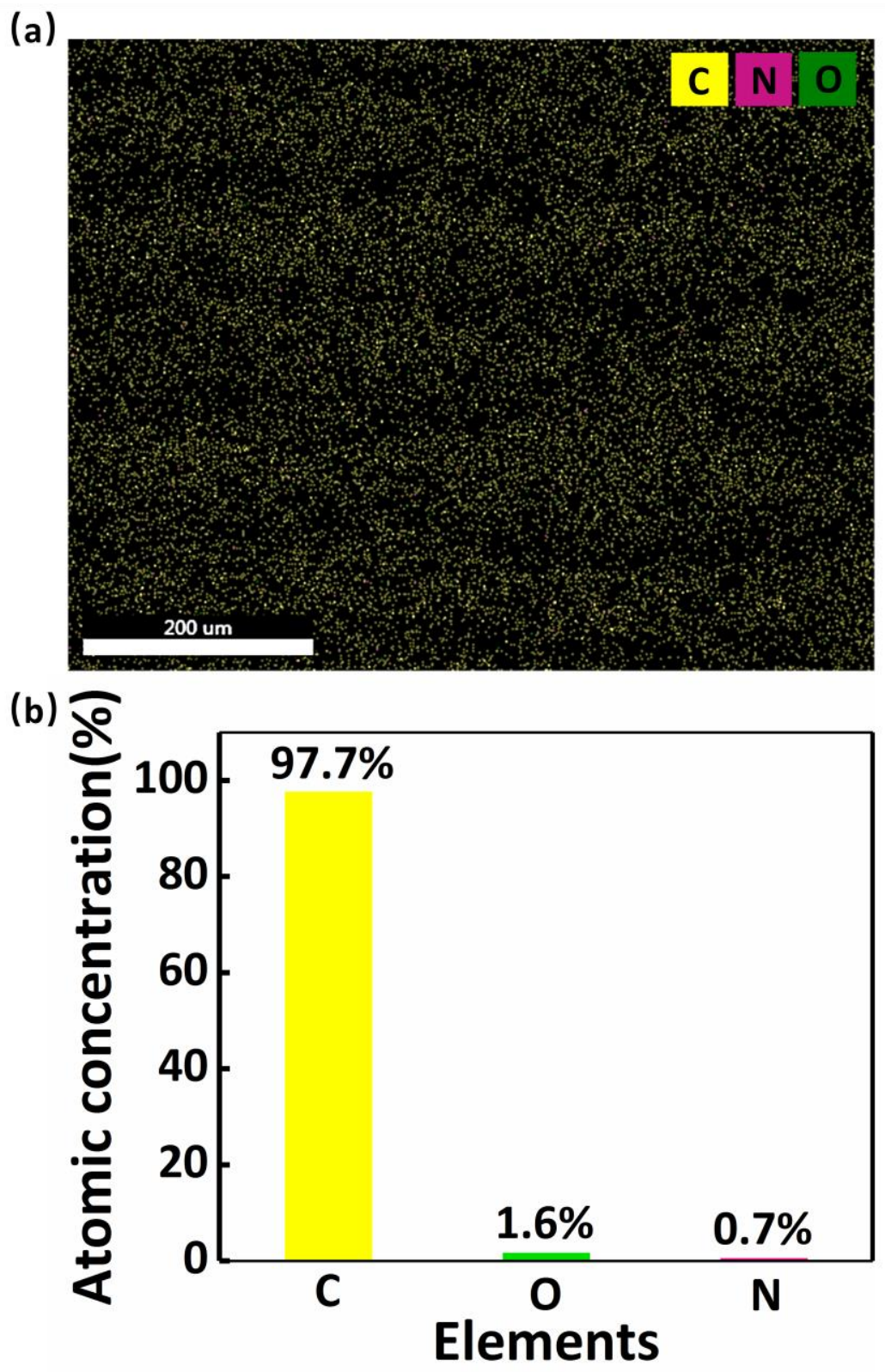

Figure S2. (a) Distribution of carbon, oxygen, and nitride for LIG on PI in the energy-dispersive X-ray spectroscopy (EDS) mapping. (b) Atomic percentage of the elements. 


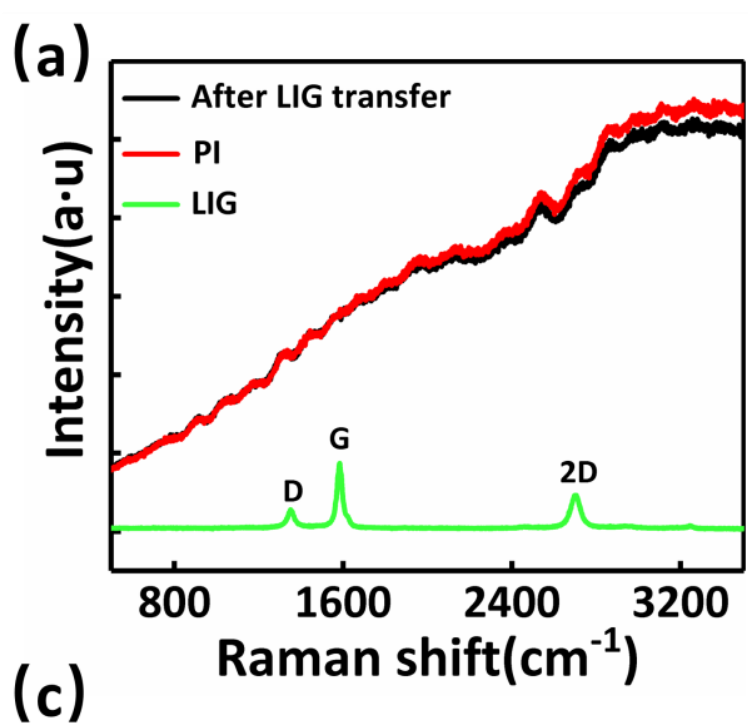

(b)
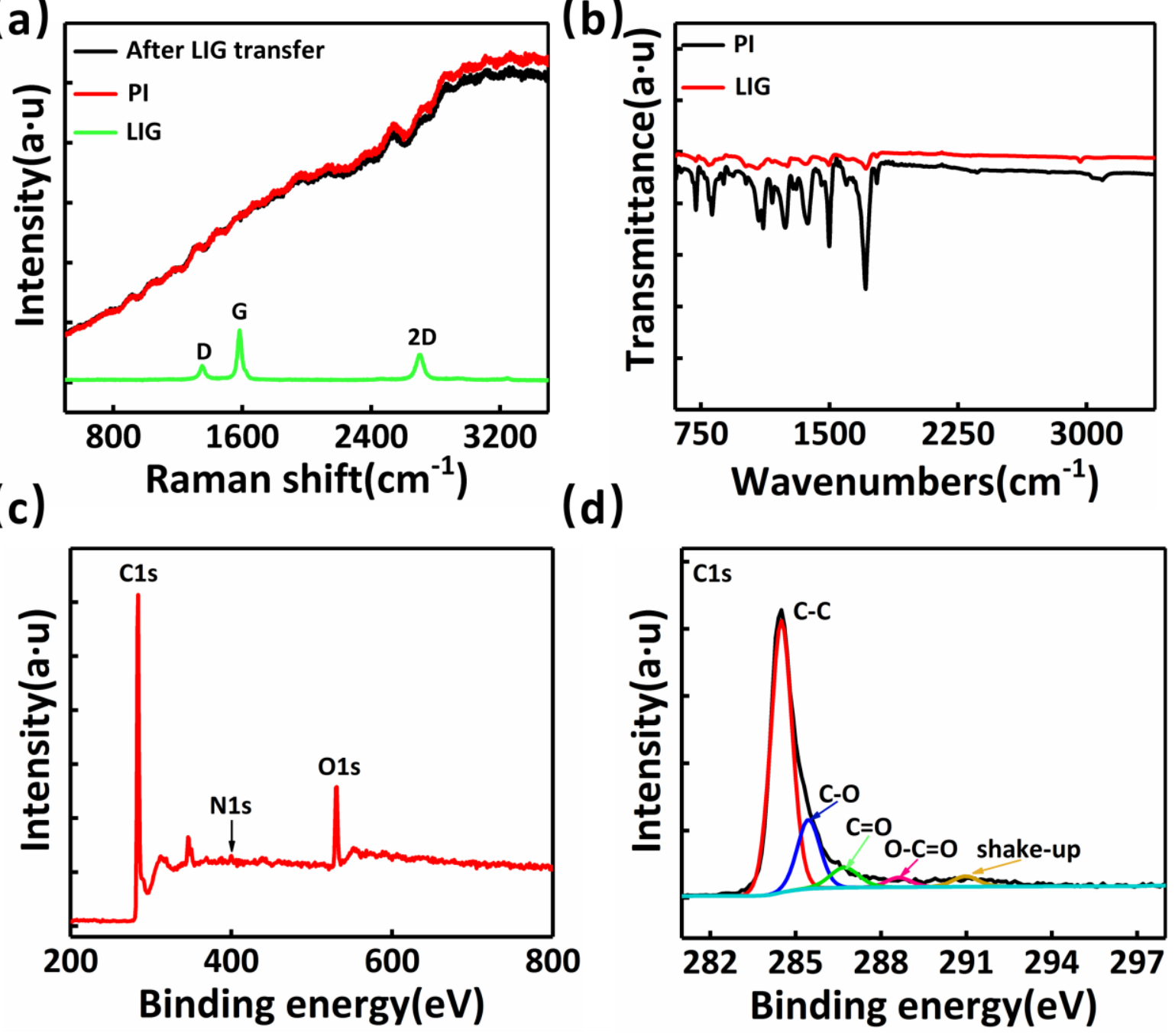

Figure S3. (a) Raman spectra for the LIG, PI, and PI after LIG transfer. (b) FTIR spectra for the LIG and PI. (c) XPS surveys of LIG on PI. (d) High-resolution XPS spectra of C1s for LIG on PI. 


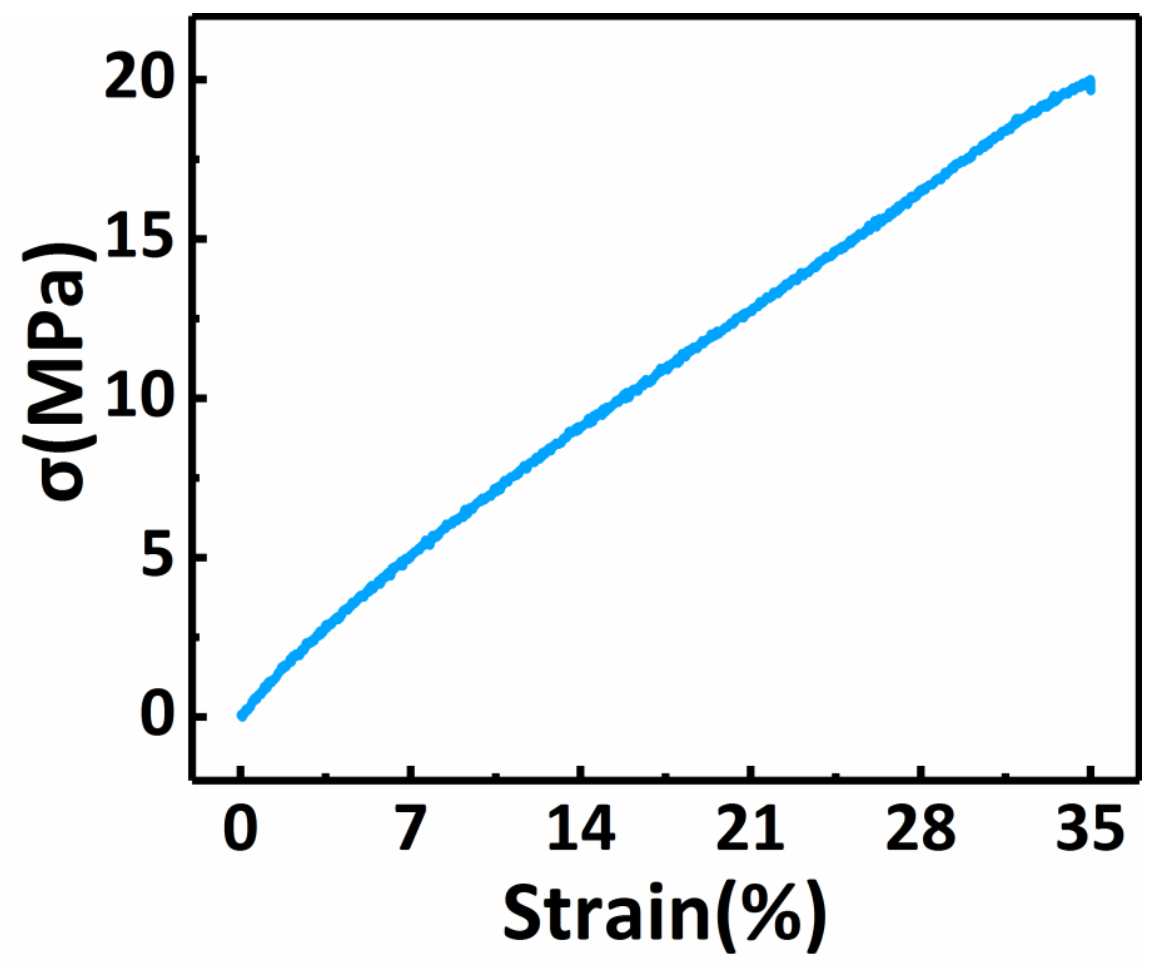

Figure S4. Stress-strain curve for LIG-based strain sensor with mesh pattern.

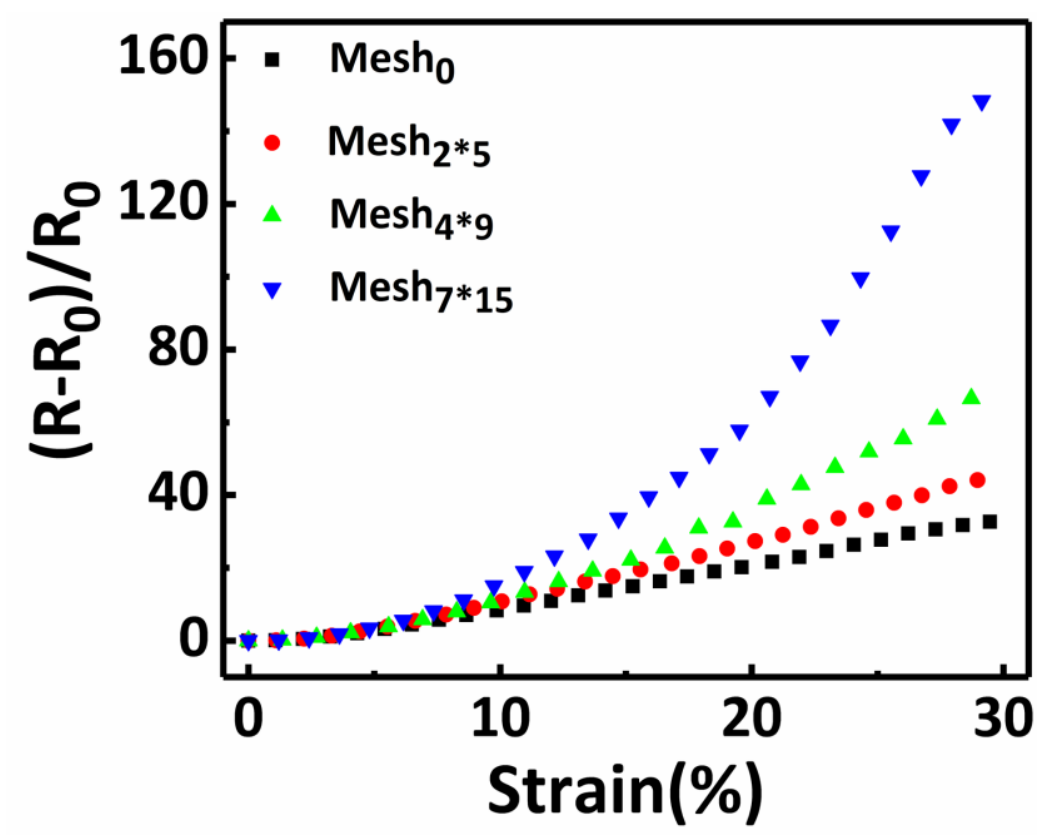

Figure S5. (a) Relative resistance change under the strain of 0-30\% for the LIG strain sensors with different mesh patterns, respectively. 


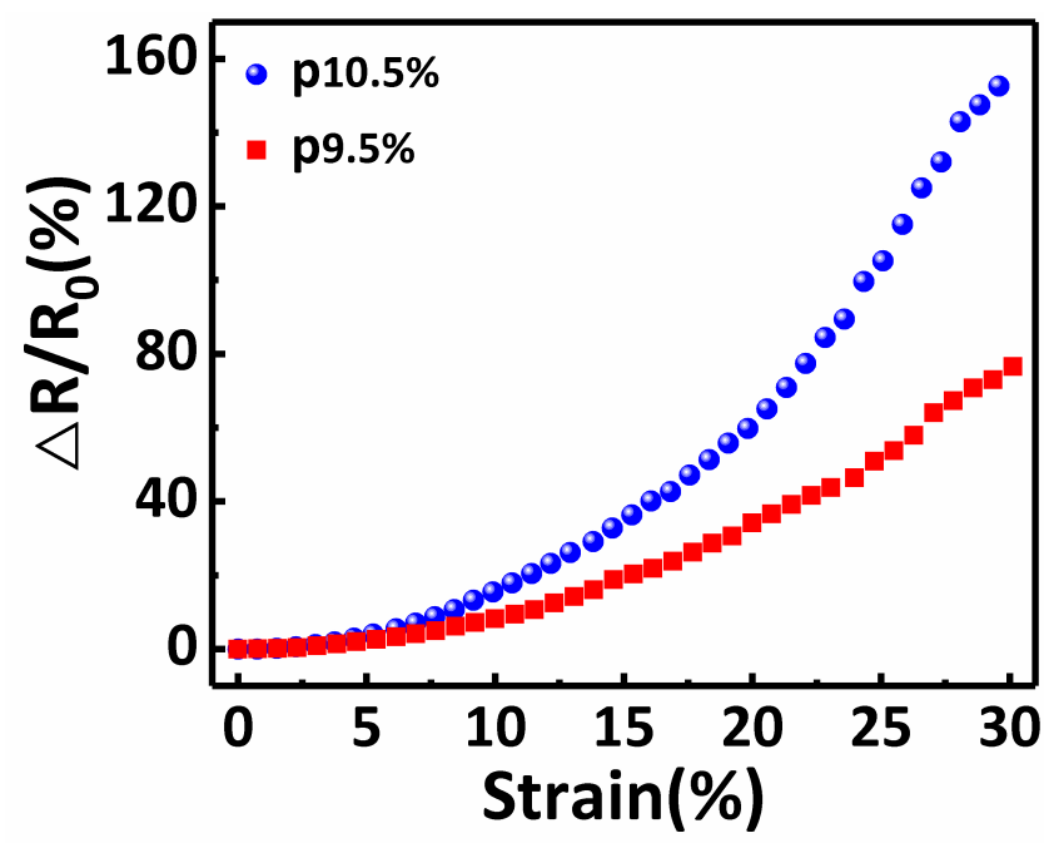

Figure S6. Relative resistance change for the LIG strain sensors with mesh patterns with different laser power of laser writing, respectively.

(a)

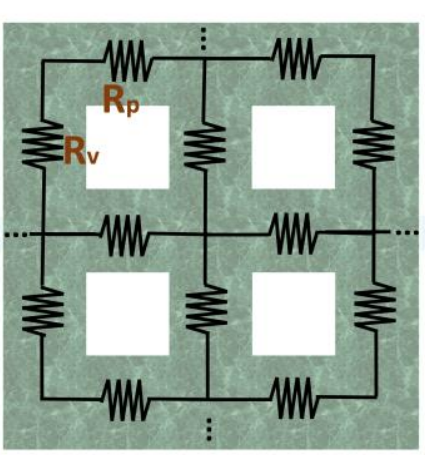

(b)

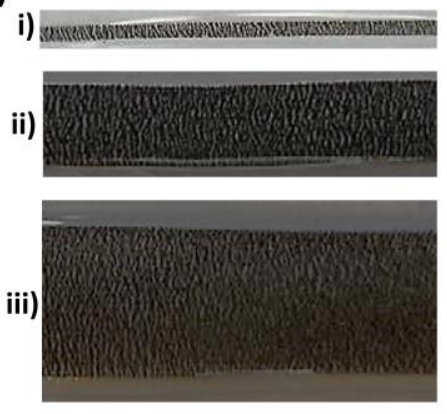

(c)

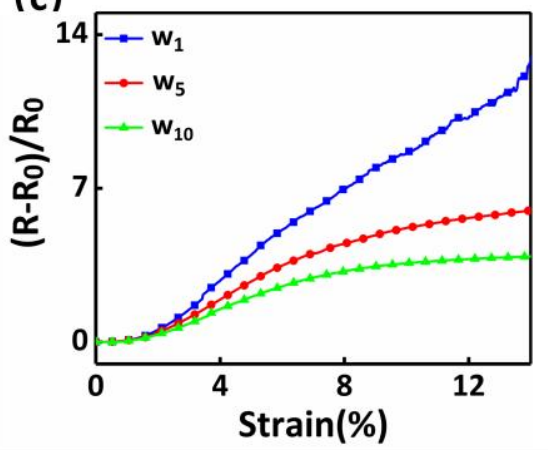

Figure S7. (a) Schematic illustration of the sensing mechanism of the strain sensor with mesh pattern. (b) Deformation photographs of nonpatterned LIG with different widths under the same tensile strain. (c) Relative resistance change under tensile strain for the nonpatterned LIG with width of 1,5 , and $10 \mathrm{~mm}$. 


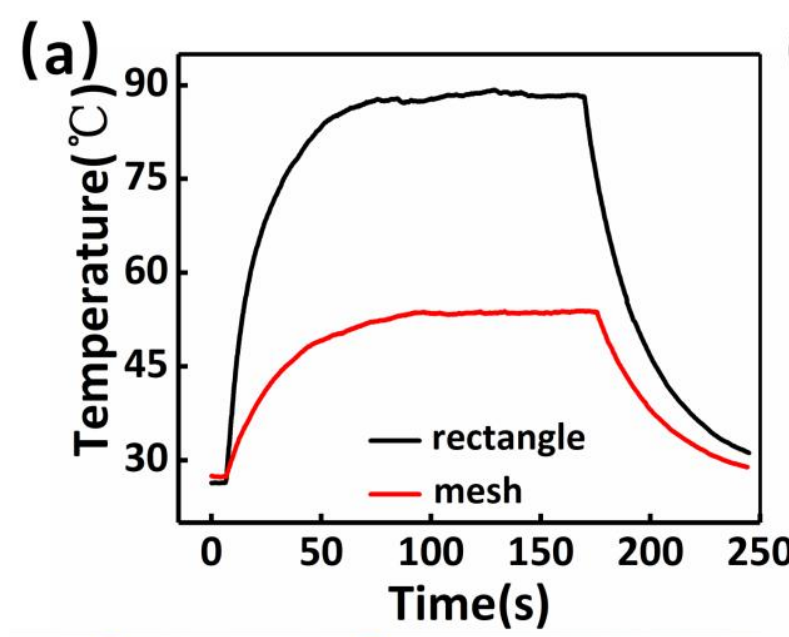

(b)
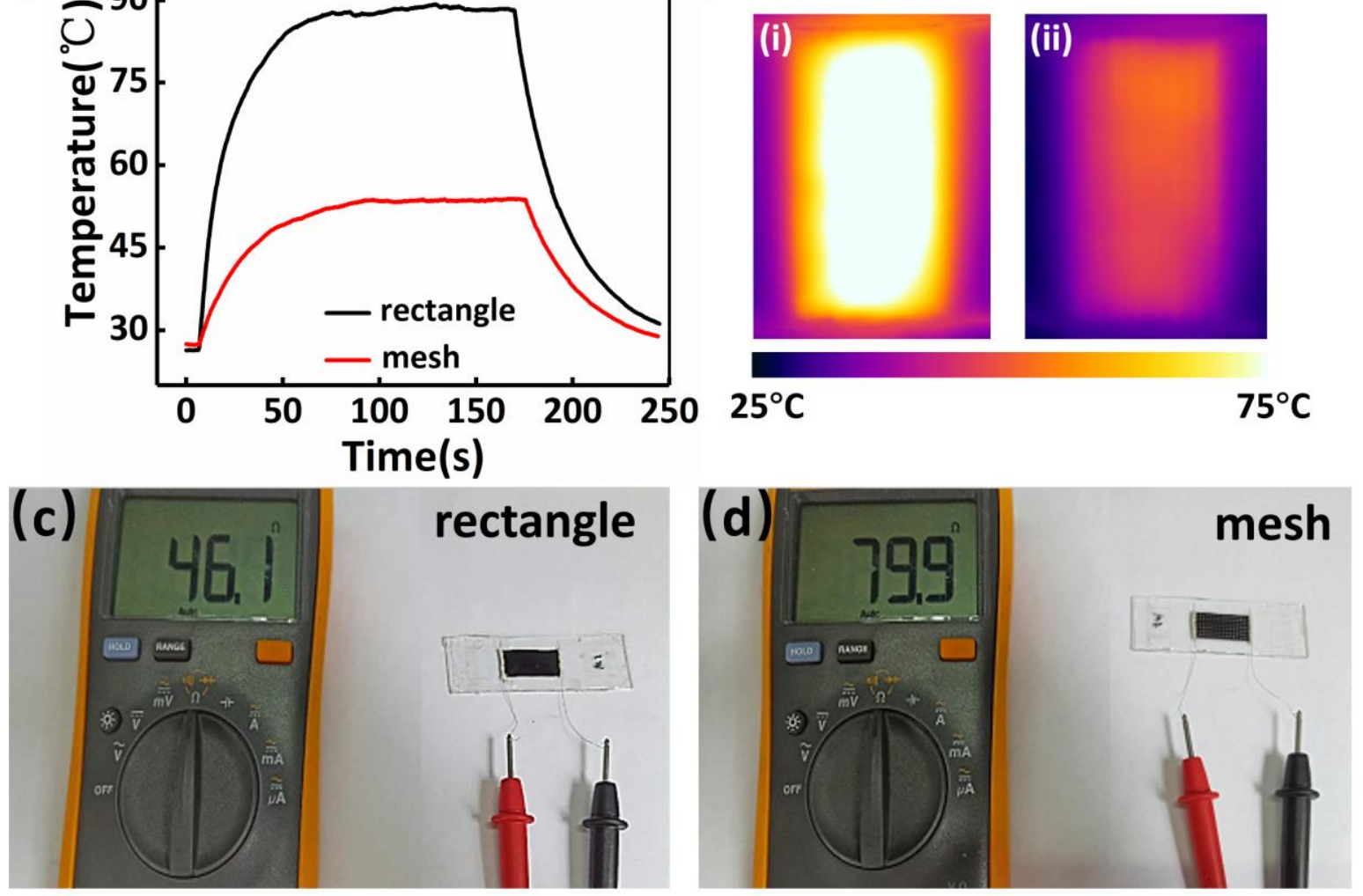

Figure S8. (a) Time-dependent surface temperatures and (b) IR camera images of the device based on LIG with rectangle and mesh patterns under the same voltage of $5 \mathrm{~V}$, respectively. Measurement of the initial resistance for (c) rectangle and $(\mathrm{d})$ mesh pattern.
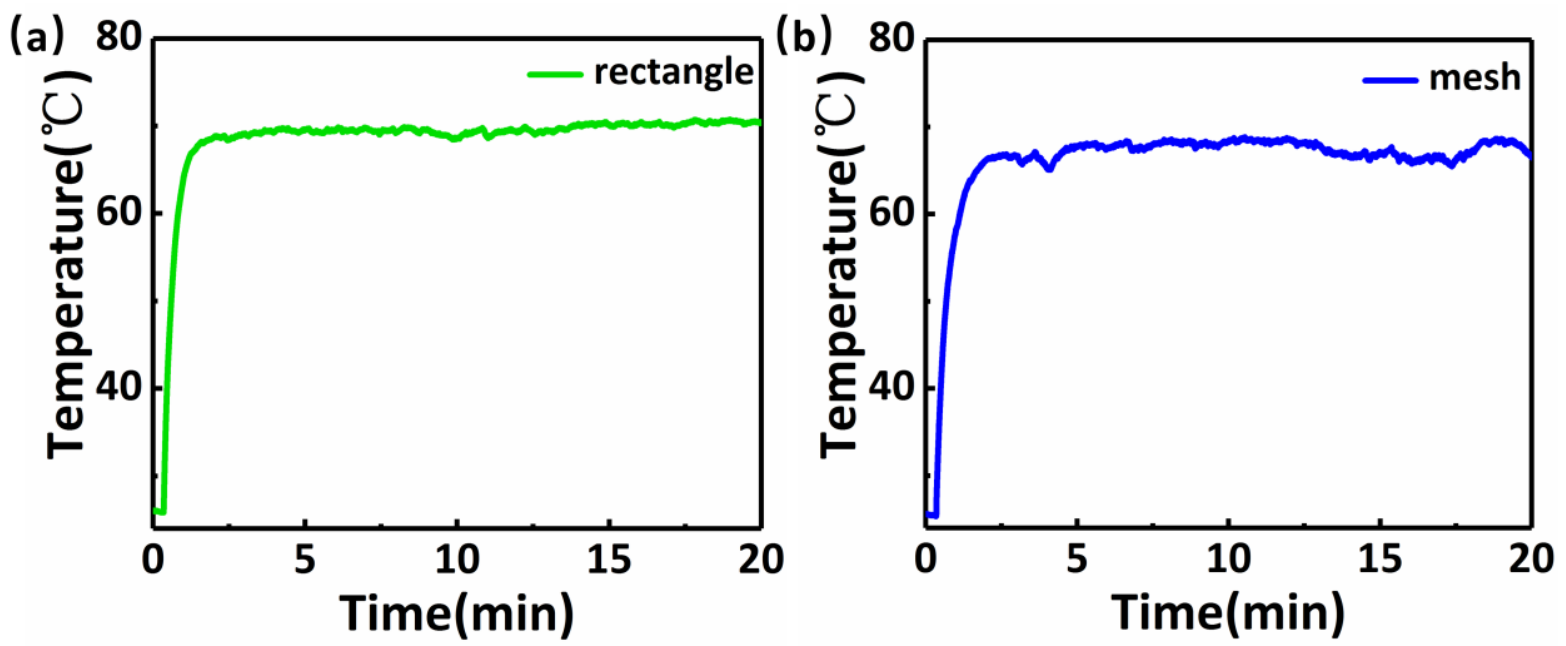

Figure S9. Long-term heating stability of the LIG-based device for (a) rectangle and (b) mesh pattern. 

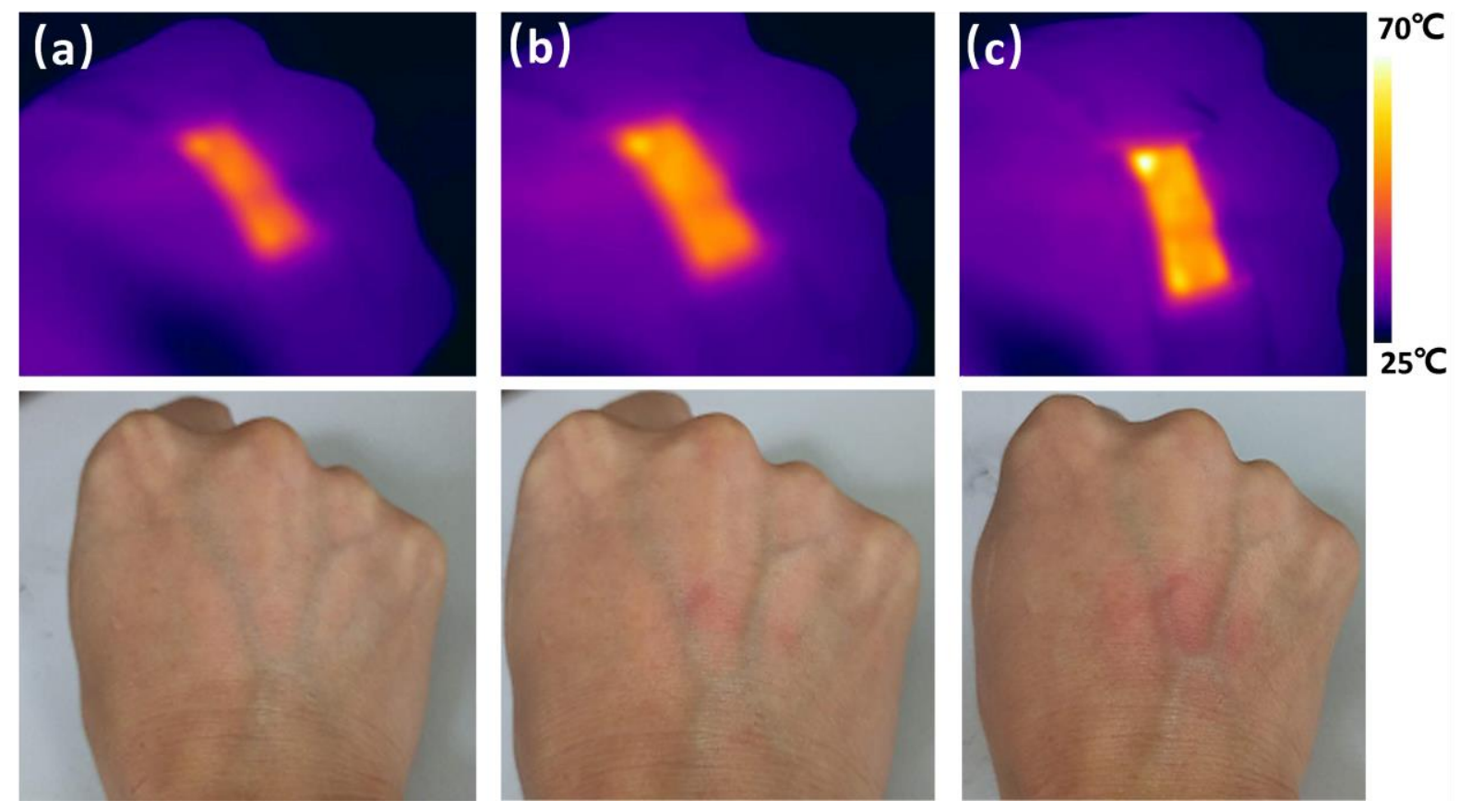

Figure S10. The effect of the heating temperature for the LIG-based heater with rectangle pattern on the skin for (a) $50{ }^{\circ} \mathrm{C}$, (b) $60{ }^{\circ} \mathrm{C}$ and (c) $70{ }^{\circ} \mathrm{C}$, respectively. 